\title{
Current Status and Effect of Outpatient Cardiac Rehabilitation After Percutaneous Coronary Intervention in Japan
}

\author{
Koshiro Kanaoka, MD; Tsunenari Soeda, MD, PhD; Satoshi Terasaki, MD; \\ Yuichi Nishioka, MD, PhD; Tomoya Myojin, MD; Shinichiro Kubo; \\ Katsuki Okada, MD, PhD; Tatsuya Noda, MD, PhD; Makoto Watanabe, MD, PhD; \\ Rika Kawakami, MD, PhD; Yasushi Sakata, MD, PhD; \\ Tomoaki Imamura, MD, PhD; Yoshihiko Saito, MD, PhD
}

\begin{abstract}
Background: Although cardiac rehabilitation (CR) has been reported to be associated with better clinical outcomes in patients with cardiovascular diseases, there are few nationwide studies about CR participation by patients with coronary artery disease in Japan.

Methods and Results: We performed a nationwide retrospective cohort study using the National Database of Health Insurance Claims and Specific Health Checkups of Japan between April 2014 and March 2018. Patients were divided into 2 groups (acute coronary syndrome $[A C S$ ] and stable coronary artery disease [SCAD]), and the rates of participation in in- and outpatient CR after percutaneous coronary intervention (PCI) were investigated. Propensity score-matched analysis was performed and the association between outpatient CR participation and all-cause mortality 3 months after PCI was examined. Overall, 616,664 patients (ACS, $\mathrm{n}=202,853$; sCAD, $\mathrm{n}=413,811$ ) were analyzed. The participation rates of CR increased annually. The participation rate was higher for inpatient than outpatient CR in both the ACS (52\% vs. $9 \%$, respectively) and SCAD (15\% vs. 3\%, respectively) groups. Prognosis was better for patients with than without outpatient $\mathrm{CR}$ in both the ACS (hazard ratio [HR] 0.52; 95\% confidence interval [CI] 0.47$0.59)$ and SCAD (HR 0.72; 95\% Cl 0.65-0.80) groups.
\end{abstract}

Conclusions: Outpatient CR was associated with a better prognosis in patients with ACS or SCAD. The participation rates of outpatient CR following PCl were extremely low in Japan.

Key Words: Cardiac rehabilitation; Coronary artery disease; Percutaneous coronary intervention; The National database

$\mathbf{C}$ ardiac rehabilitation $(\mathrm{CR})$ is an evidence-based intervention associated with a reduction in mortality and rehospitalization, as well as improved quality of life, in patients with coronary artery disease (CAD), including both acute coronary syndrome (ACS) and stable CAD (sCAD). ${ }^{1-3}$ Current guidelines in Japan show that $\mathrm{CR}$ is strongly recommended after myocardial infarction and percutaneous coronary intervention (PCI). ${ }^{4}$

However, the rate of CR participation is low worldwide, especially in Japan. 5,6 Previous surveys demonstrated that the rates of participation in CR for ACS in Japan were lower than in the US, UK, and France..$^{7,8}$ A recent Japanese survey (i.e., Japanese Registry Of All cardiac and vascular Diseases [JROAD]) showed that the overall number of patients receiving CR is gradually increasing in Japan, ${ }^{9}$ but very little evidence has been published regarding CR participation rates and the effect of CR on mortality after PCI.

The aims of this study were to describe the current participation rates of CR after PCI in ACS and sCAD patients and to investigate the effects of outpatient $\mathrm{CR}$ after PCI on the mortality rate using a nationwide administrative database in Japan.

\section{Data Source}

Data were extracted from the National Database of Health Insurance Claims and Specific Health Checkups of Japan (NDB) between April 2013 and March 2019; data were

Received January 20, 2021; accepted January 20, 2021; J-STAGE Advance Publication released online February 27, 2021 Time for primary review: 1 day

Department of Cardiovascular Medicine (K.K., T.S., S.T., M.W., R.K., Y. Saito), Department of Public Health, Health Management and Policy (Y.N., T.M., S.K., T.N., T.I.), Nara Medical University, Kashihara; Department of Cardiovascular Medicine, Osaka University Graduate School of Medicine, Osaka (K.O., Y. Sakata), Japan

Mailing address: Yoshihiko Saito, MD, PhD, Department of Cardiovascular Medicine, Nara Medical University, 840 Shijo-cho, Kashihara 634-8521, Japan. E-mail: yssaito@naramed-u.ac.jp

All rights are reserved to the Japanese Circulation Society. For permissions, please e-mail: cr@j-circ.or.jp

ISSN-2434-0790 


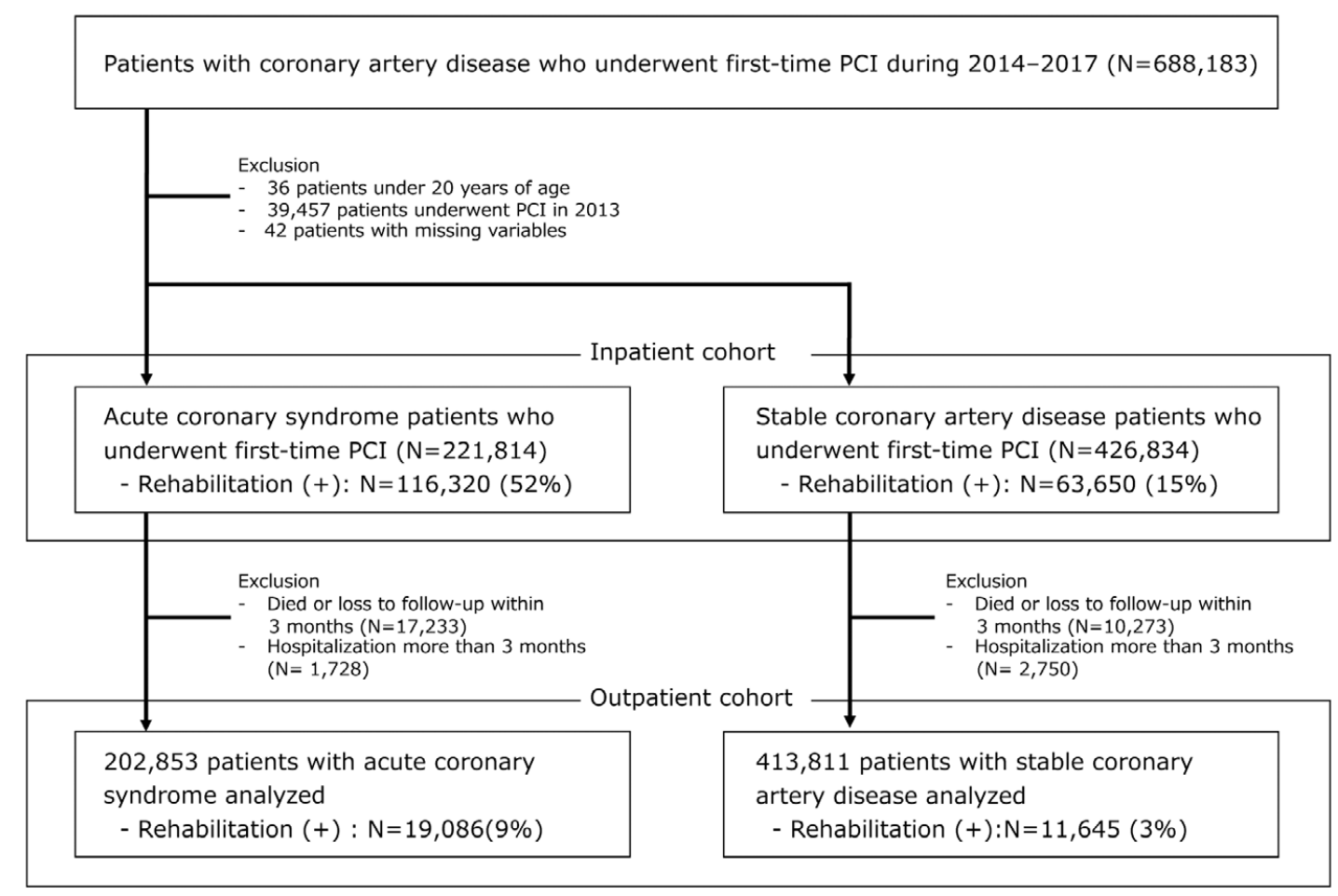

Figure 1. Flowchart depicting the study design. In all, 202,853 patients with acute coronary syndrome and 413,811 patients with stable coronary disease were analyzed as the outpatient cohort. PCl, percutaneous coronary intervention.

provided by the Japan Ministry of Health, Labour, and Welfare. The NDB is a Japanese administrative claims database that includes claims on inpatient or outpatient services and prescriptions. Japan has a universal health coverage system, and the NDB covers approximately $98 \%$ of data on healthcare services provided by healthcare institutions, regardless of the type of insurance. ${ }^{10}$ The NDB allows each patient to be followed longitudinally using an individual claims data system and a patient-matching technique in the NDB. ${ }^{11,12}$ The NDB contains the following information: (1) sex; (2) age group (5-year age groups); (3) diagnostic codes based on the 10th revision of the International Statistical Classification of Diseases (ICD-10) codes and outcome categories; and (4) codes for medical care received, drugs prescribed, and medical examinations performed, not including tests results.

\section{Study Design and Setting}

The present retrospective cohort study was based on the NDB. Data were extracted for patients who underwent PCI and were diagnosed with CAD based on ICD-10 codes between April 2014 and March 2018. To extract only patients who underwent their first PCI procedure, a 1-year interval (April 2013-March 2014) was considered as the washout period, and patients who underwent PCI between April 2013 and March 2014 were excluded. Patients <20 years of age or those with missing data were also excluded.

Of the 688,183 patients who underwent PCI between April 2014 and March 2018, 648,648 with CAD underwent PCI for the first time, and patients eligible for inpatient CR were identified. There were 221,814 patients with ACS and
426,834 with sCAD (inpatient cohort). Of these, 202,853 patients with ACS and 413,811 patients with sCAD were alive 3 months after discharge, were followed-up for more than 3 months and were evaluated as an outpatient cohort (Figure 1).

First, patient participation rates in inpatient and outpatient CR were evaluated. Patients were divided into 2 groups (i.e., ACS and sCAD groups). The inpatient CR participation rate was defined as the number of inpatient CR participants divided by the total number of hospitalized patients; the outpatient CR participation rate was defined as the number of outpatient CR participants divided by the number of patients who were discharged alive and followed-up for more than 3 months. The CR participation rates according to $\mathrm{CAD}$ were described according to year and prefecture.

Second, we examined the association between outpatient CR participation rate and primary and secondary outcomes (see below) according to CAD category using propensity score matching.

\section{Variable Definitions}

Data were obtained from the NDB regarding the age, sex, date of first PCI, disease codes, prescribed medications and medical treatments for all patients. The patients were classified as having ACS or SCAD based on the procedure codes for PCI (i.e., ACS was defined as PCI for acute myocardial infarction or unstable angina, whereas sCAD was defined as PCI for stable CAD). Inpatient CR participation was identified as $\geq 1$ procedure code for $C R$ during the index hospitalization. In Japan, outpatient CR is 


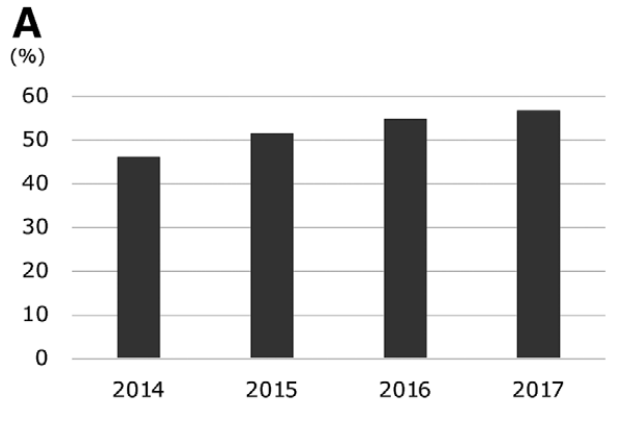

Inpatient rehabilitation for ACS
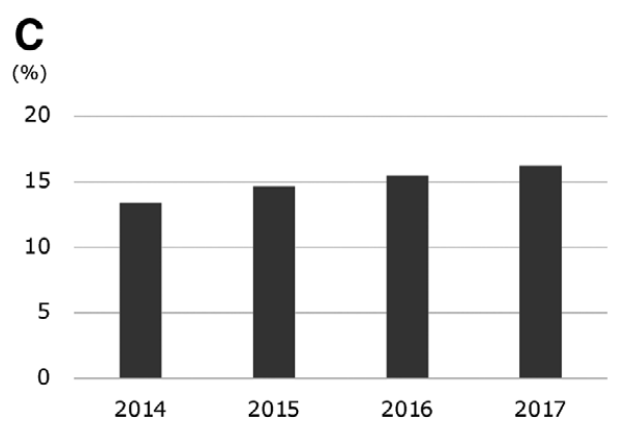

Inpatient rehabilitation for $\mathrm{SCAD}$

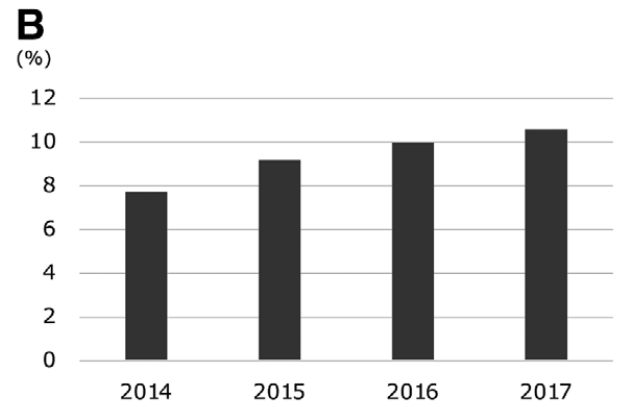

Outpatient rehabilitation for ACS

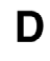

(\%)

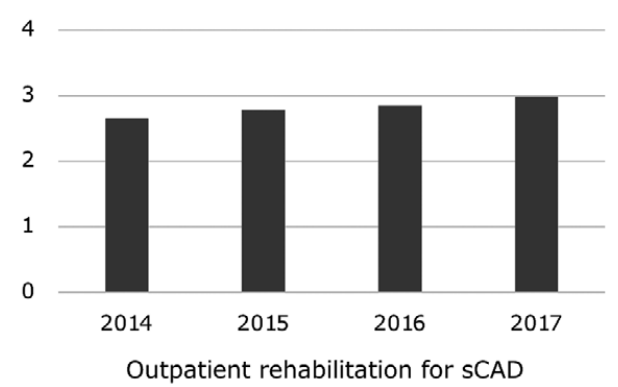

Figure 2. (A,C) Inpatient and (B,D) outpatient cardiac rehabilitation participation rates among acute coronary syndrome (ACS; A,B) or stable coronary artery disease (SCAD; C,D) patients between 2014 and 2017. Overall cardiac rehabilitation participation rates increased gradually from 2014 to 2017 . The prescription rate for outpatient cardiac rehabilitation was lower than that for inpatient cardiac rehabilitation.

covered by insurance up to 60 min per session and 3 times a week. Outpatient CR participation was identified as $\geq 1$ procedure code for CR between discharge and 3 months after the first PCI. Patient characteristics were obtained from inpatient and outpatient data 3 months before hospitalization.

\section{Outcomes}

The primary outcome in the present study was all-cause mortality. Death in the database was defined based on the logic-based method for determining mortality (sensitivity, 94.2\%; specificity, 98.8\%). ${ }^{13}$ Secondary outcomes were unexpected PCI for ACS, planned PCI, hospitalization for acute heart failure, gastrointestinal bleeding with endoscopic or operative hemostasis, and cerebrovascular infarction and intracranial hemorrhage. Hospitalization for acute heart failure was defined as an emergency hospitalization with heart failure treated with intravenous diuretics. Cerebrovascular infarction and intracranial hemorrhage were defined as hospitalization into the stroke care unit, surgery, or catheter intervention for cerebral hemorrhage or stroke, or the use of alteplase or edaravone with the codes of stroke.

\section{Statistical Analysis}

Patient clinical characteristics according to outpatient CR participation before and after matching are described as numbers and percentages for categorical variables or as the median and interquartile range (IQR) for continuous variables. Baseline characteristics were compared using the standardized difference. Propensity scores were generated using multivariate logistic regression models for participation in outpatient CR. Variables included in the model were age, sex, medications, emergency admission, treatments during the index hospitalization, and length of hospital stay. Propensity score matching was performed for $\mathrm{CR}$ participants and non-participants in a $1: 1$ ratio using nearest neighbor matching without replacement. Kaplan-Meier curves, log-rank tests and Cox proportional hazards models were used to estimate the association between outpatient CR participation and each endpoint. Survival time was counted from the first PCI date with a minimum of 90 days (landmark period). In addition, sensitivity analyses were conducted for the primary outcome. First, we performed the same analysis using multivariable Cox regression analysis using the same covariates instead of propensity score matching. Second, to analyze the effect of outpatient CR in patients who underwent inpatient $\mathrm{CR}$, propensity score matching analysis was performed in patients who underwent CR during the first hospitalization. Data were managed using structured query language (SQL), and statistical analyses were performed using STATA v15 (Stata Corp., College Station, TX, USA). Two-tailed $\mathrm{P}<0.05$ was considered significant.

\section{Ethics Statement}

The study protocol was approved by the Ethics Committee of Nara Medical University (Registration no. 1123-2) and 
A

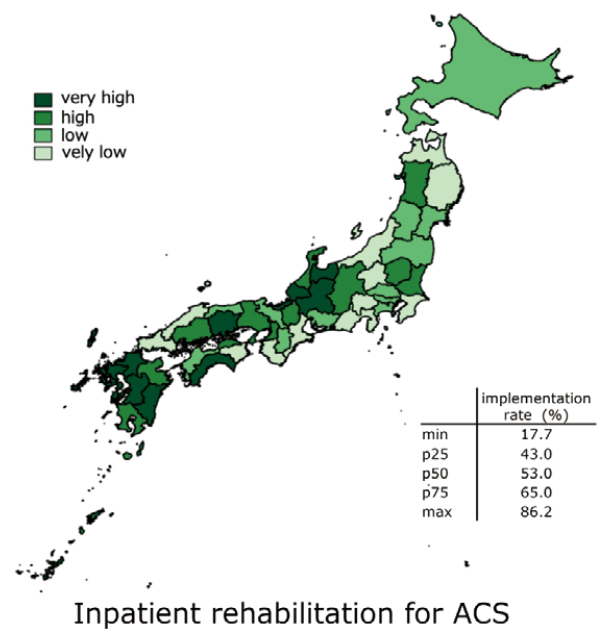

C

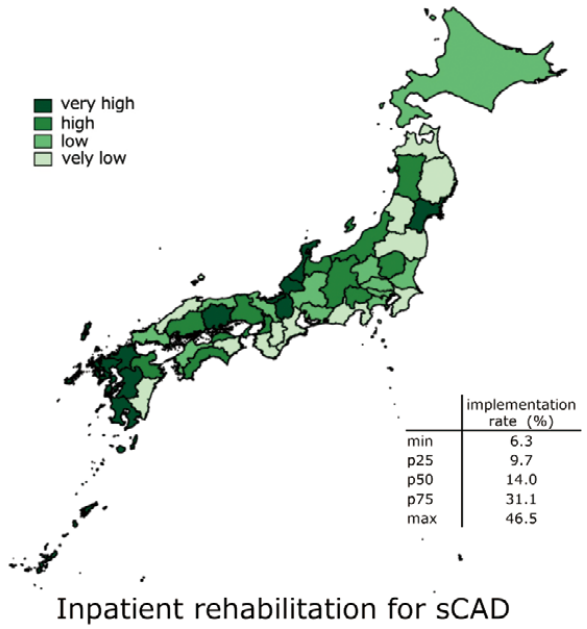

B

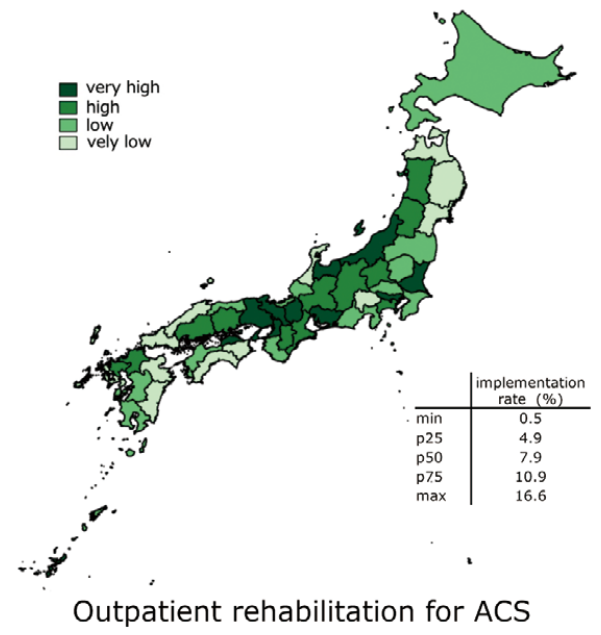

D

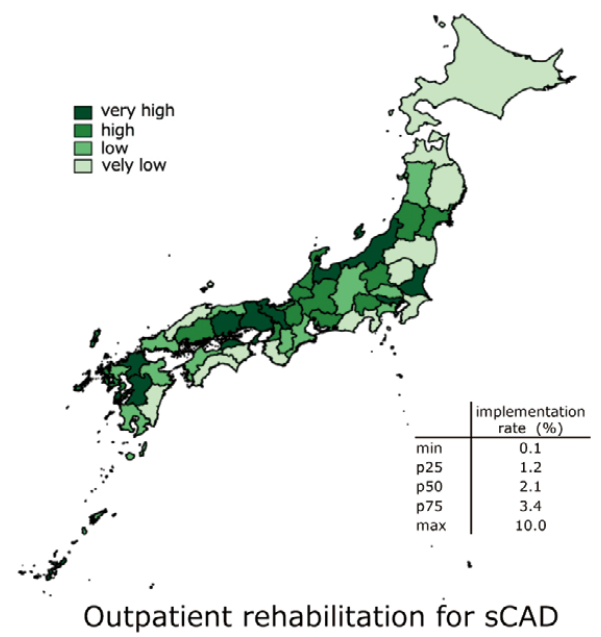

Figure 3. (A,C) Inpatient and (B,D) outpatient cardiac rehabilitation participation rates among acute coronary syndrome (ACS; $\mathbf{A}, \mathbf{B})$ or stable coronary artery disease (SCAD; C,D) patients according to prefecture. The inpatient and outpatient participation rates varied widely between prefectures.

the study was conducted according to the principles of the Declaration of Helsinki. The requirement for individual informed consent was waived because all data were originally anonymized when provided by the Ministry of Health, Labour and Welfare of Japan.

\section{Results}

\section{Inpatient and Outpatient CR Participation}

Of the hospitalized patients with CAD who underwent their first PCI, $52 \%$ of ACS patients $(n=116,320)$ and $15 \%$ of sCAD patients $(n=63,650)$ participated in inpatient CR. Among the population eligible for outpatient CR, 9\% of ACS patients $(n=19,086)$ and $3 \%$ of sCAD patients $(n=11,645)$ participated in outpatient CR. Of the patients who underwent inpatient $\mathrm{CR}$, the median number of $\mathrm{CR}$ sessions within 3 months after the first PCI was 7 (IQR 3-15) for ACS and 6 (IQR 2-12) for stable CAD. The proportion of patients taking part in CR increased between 2014 and 2017. However, the proportion was relatively low for outpatient compared with inpatient CR (Figure 2). The inpatient and outpatient participation rates varied widely across prefectures, ranging from $17.7 \%$ to $86.2 \%$ for inpatient CR and from $0.5 \%$ to $16.6 \%$ for outpatient CR among ACS patients, and from $6.3 \%$ to $46.5 \%$ for inpatient and from $0.1 \%$ to $10.0 \%$ for outpatient CR among sCAD patients (Figure 3).

\section{Patient Characteristics}

The characteristics of the patients who participated in outpatient CR are presented in Tables 1 and 2. Most patients were $71-80$ years of age $(58,990$ [29.1\%] and $157,411[38.0 \%$ ] patients in the ACS and sCAD groups, respectively), and the prevalence of females in both groups was $<30 \%$. The proportion of patients $<80$ years of age, using $\beta$-blockers, angiotensin-converting enzyme inhibitors, angiotensin receptor blockers, statins, or respirators, those requiring mechanical circulatory support, and those taking part in CR during hospitalization was higher for patients who took part in outpatient CR than those who did not. 
Table 1. Characteristics of Patients With ACS Before and After Propensity Score Matching

\begin{tabular}{|c|c|c|c|c|c|c|c|}
\hline & \multirow{2}{*}{ All patients } & \multicolumn{3}{|c|}{ Before matching } & \multicolumn{3}{|c|}{ After matching } \\
\hline & & CR (-) & CR (+) & |SD & CR (-) & CR (+) & |SD| \\
\hline No. patients & 202,853 & 183,767 & 19,086 & & 19,086 & 19,086 & \\
\hline \multicolumn{8}{|l|}{ Age group (years) } \\
\hline$<51$ & $17,069(8)$ & $14,946(8)$ & $2,123(11)$ & 0.295 & $2,012(11)$ & $2,123(11)$ & 0.02 \\
\hline $51-60$ & $28,356(14)$ & $25,130(14)$ & $3,226(17)$ & & $3,179(17)$ & $3,226(17)$ & \\
\hline $61-70$ & $54,532(27)$ & $48,534(26)$ & $5,998(31)$ & & $5,964(31)$ & $5,998(31)$ & \\
\hline $71-80$ & $58,990(29)$ & $53,197(29)$ & $5,793(30)$ & & $5,989(31)$ & $5,793(30)$ & \\
\hline $81-90$ & $38,409(19)$ & $36,532(20)$ & $1,877(10)$ & & $1,887(10)$ & $1,877(10)$ & \\
\hline$>90$ & $5,497(3)$ & $5,428(3)$ & $69(0.4)$ & & $55(0.3)$ & $69(0.4)$ & \\
\hline Female sex & $48,774(24)$ & $45,029(25)$ & $3,745(20)$ & 0.118 & $3,746(20)$ & $3,745(20)$ & $<0.001$ \\
\hline \multicolumn{8}{|l|}{$\begin{array}{l}\text { Medications before or } \\
\text { during hospitalization }\end{array}$} \\
\hline$\beta$-blockers & $129,287(64)$ & $115,032(63)$ & $14,255(75)$ & 0.263 & $14,164(74)$ & $14,255(75)$ & 0.011 \\
\hline ACEI/ARBs & $155,162(77)$ & $139,453(76)$ & $15,709(82)$ & 0.158 & $15,804(83)$ & $15,709(82)$ & 0.013 \\
\hline CCBs & $95,251(47)$ & $87,323(48)$ & $7,928(42)$ & 0.121 & $7,955(42)$ & $7,928(42)$ & 0.003 \\
\hline MRA & $26,786(13)$ & $23,978(13)$ & $2,808(15)$ & 0.048 & $2,567(13)$ & $2,808(15)$ & 0.036 \\
\hline Antidiabetes agents & $64,825(32)$ & $59,121(32)$ & $5,704(30)$ & 0.049 & $5,597(29)$ & $5,704(30)$ & 0.012 \\
\hline PPIs & $180,229(89)$ & $162,699(89)$ & $17,530(92)$ & 0.112 & $17,560(92)$ & $17,530(92)$ & 0.006 \\
\hline Statins & $173,810(86)$ & $156,235(85)$ & $17,575(92)$ & 0.223 & $17,604(92)$ & $17,575(92)$ & 0.006 \\
\hline Loop diuretics & $43,978(22)$ & $40,263(22)$ & $3,715(20)$ & 0.060 & $3,450(18)$ & $3,715(20)$ & 0.036 \\
\hline COPD/asthma medications & $13,993(6.9)$ & $12,797(7.0)$ & $1,196(6.3)$ & 0.028 & $1,076(5.6)$ & $1,196(6.3)$ & 0.027 \\
\hline End-stage renal disease & $3,632(1.8)$ & $3,537(1.9)$ & $95(0.5)$ & 0.131 & $78(0.4)$ & $95(0.5)$ & 0.013 \\
\hline Emergency admission & $190,351(94)$ & $171,906(94)$ & $18,445(97)$ & 0.144 & $18,538(97)$ & $18,445(97)$ & 0.028 \\
\hline \multicolumn{8}{|l|}{$\begin{array}{l}\text { Treatments during } \\
\text { hospitalization }\end{array}$} \\
\hline NPPV or respirator use & $16,051(8)$ & $14,444(7.9)$ & $1,607(8.4)$ & 0.020 & $1,369(7.2)$ & $1,607(8.4)$ & 0.047 \\
\hline IABP & $20,898(10)$ & $18,402(10)$ & $2,496(13)$ & 0.096 & $2,161(11)$ & $2,496(13)$ & 0.054 \\
\hline ECMO & $1,320(0.7)$ & $1,117(0.6)$ & $203(1.1)$ & 0.050 & $164(0.9)$ & $203(1.1)$ & 0.021 \\
\hline \multicolumn{8}{|l|}{$\begin{array}{l}\text { No. stents implanted at first } \\
\text { PCl }\end{array}$} \\
\hline 0 & $14,039(6.9)$ & $12,895(7.0)$ & $1,144(6.0)$ & 0.008 & $1,039(5.4)$ & $1,144(6.0)$ & 0.007 \\
\hline 1 & $142,873(70)$ & $129,072(70)$ & $13,801(72)$ & & $14,043(74)$ & $13,801(72)$ & \\
\hline 2 & $37,637(19)$ & $34,200(19)$ & $3,437(18)$ & & $3,350(18)$ & $3,437(18)$ & \\
\hline$\geq 3$ & $8,304(4.1)$ & $7,600(4.1)$ & 704 (3.7) & & $654(3.4)$ & 704 (3.7) & \\
\hline CR during hospitalization & $110,559(55)$ & $93,099(51)$ & $17,460(92)$ & 1.008 & $17,459(92)$ & $17,460(92)$ & $<0.001$ \\
\hline Hospital LOS (days) & $13[8-17]$ & $12[8-17]$ & $14[10-18]$ & 0.045 & $14[10-17]$ & $14[10-18]$ & 0.021 \\
\hline
\end{tabular}

Unless indicated otherwise, data are presented as $\mathrm{n}(\%)$ or the median [interquartile range]. ACEl, angiotensin-converting-enzyme inhibitor; ACS, acute coronary syndrome; ARB, angiotensin II receptor blocker; CCBs, calcium channel blockers; COPD, chronic obstructive pulmonary disease; CR, cardiac rehabilitation; ECMO, extracorporeal membrane oxygenation; IABP, intra-aortic balloon pumping; LOS, length of stay; MRA, mineralocorticoid antagonists; NPPV, non-invasive positive pressure ventilation; $\mathrm{PCl}$, percutaneous coronary intervention; PPIs, proton pump inhibitors; SD, standardized difference.

Before matching, there were fewer female patients and patients with end-stage renal disease in the CR than nonCR group.

\section{Primary and Secondary Outcomes}

The crude primary and secondary outcomes are described in Supplementary Tables $\mathbf{1}$ and $\mathbf{2}$. After propensity score matching, 19,086 ACS patients and 11,644 sCAD patients were matched with patients who did not participate in outpatient CR. The C-statistic for the model was 0.76 and 0.76 in the ACS and sCAD groups, respectively. The baseline covariates were well balanced between the 2 groups (standardized differences $<10 \%$ for all variables). In the matched outpatient cohort, the median follow-up duration was 2.6 and 2.7 years in the ACS and SCAD groups, respectively. During follow-up of ACS patients, all-cause deaths occurred in $423(2.2 \%)$ patients with outpatient CR and 807 (4.2\%) patients without outpatient $\mathrm{CR}$. Among sCAD patients, all-cause deaths occurred in $623(5.4 \%)$ and $844(7.3 \%)$ patients among those with and without outpatient CR, respectively. Compared with non-CR participants, there was a decreased risk of all-cause mortality among CR participants in both the ACS (hazard ratio [HR] 0.52; 95\% confidence interval [CI] 0.47-0.59; $\mathrm{P}<0.001)$ and $\mathrm{sCAD}$ (HR 0.72; 95\% CI 0.65-0.80; $\mathrm{P}<0.001$ ) groups (Table 3; Figure 4).

HRs for the secondary outcomes are presented in Table 3. Participating in CR was associated with a lower risk of acute heart failure in both the ACS (HR 0.67; 95\% CI 0.58-0.78; P<0.001) and SCAD (HR 0.76; 95\% CI 0.67$0.87 ; \mathrm{P}<0.001)$ groups and tended to be associated with a lower risk of emergency PCI in the ACS group (HR 0.86; 
Table 2. Characteristics of Patients With SCAD Before and After Propensity Score Matching

\begin{tabular}{|c|c|c|c|c|c|c|c|}
\hline & \multirow{2}{*}{ All patients } & \multicolumn{3}{|c|}{ Before matching } & \multicolumn{3}{|c|}{ After matching } \\
\hline & & CR (-) & CR (+) & |SD| & CR (-) & CR (+) & $|\mathrm{SD}|$ \\
\hline No. patients & 413,811 & 402,166 & 11,645 & & 11,644 & 11,644 & \\
\hline \multicolumn{8}{|l|}{ Age (years) } \\
\hline$<51$ & $14,684(3.5)$ & $14,128(3.5)$ & $556(4.8)$ & 0.127 & $472(4.1)$ & $555(4.8)$ & 0.05 \\
\hline $51-60$ & 38,224 (9.2) & $37,006(9.2)$ & $1,218(11)$ & & $1,152(9.9)$ & $1,218(11)$ & \\
\hline $61-70$ & $109,571(27)$ & $106,334(26)$ & 3,237 (28) & & $3,170(27)$ & $3,237(28)$ & \\
\hline $71-80$ & $157,411(38)$ & $152,848(38)$ & $4,563(39)$ & & $4,632(40)$ & $4,563(39)$ & \\
\hline $81-90$ & $87,808(21)$ & $85,817(21)$ & $1,991(17)$ & & $2,154(19)$ & $1,991(17)$ & \\
\hline$>91$ & $6,113(1.5)$ & $6,033(1.5)$ & $80(0.7)$ & & $64(0.5)$ & $80(0.7)$ & \\
\hline Female sex & $107,572(26)$ & $104,488(26)$ & $3,084(27)$ & 0.011 & $3,161(27)$ & $3,084(27)$ & 0.015 \\
\hline \multicolumn{8}{|l|}{$\begin{array}{l}\text { Medications before or } \\
\text { during hospitalization }\end{array}$} \\
\hline$\beta$-blockers & $198,331(48)$ & $191,648(48)$ & $6,683(57)$ & 0.196 & $6,600(57)$ & $6,682(57)$ & 0.014 \\
\hline ACEI/ARBs & $247,411(60)$ & $239,666(60)$ & $7,745(67)$ & 0.144 & $7,719(66)$ & $7,744(67)$ & 0.005 \\
\hline CCBs & $237,640(57)$ & $231,457(58)$ & $6,183(53)$ & 0.090 & $6,253(54)$ & $6,183(53)$ & 0.012 \\
\hline MRA & $39,753(9.6)$ & $37,819(9.4)$ & $1,934(17)$ & 0.215 & $1,843(16)$ & $1,933(17)$ & 0.021 \\
\hline Antidiabetes agents & $155,963(38)$ & $151,648(38)$ & $4,315(37)$ & 0.014 & $4,178(36)$ & $4,315(37)$ & 0.024 \\
\hline PPIs & $306,637(74)$ & $297,648(74)$ & $8,989(77)$ & 0.074 & $8,960(77)$ & $8,988(77)$ & 0.006 \\
\hline Statins & $278,769(67)$ & $270,246(67)$ & $8,523(73)$ & 0.131 & $8,511(73)$ & $8,522(73)$ & 0.002 \\
\hline Loop diuretics & $81,998(20)$ & 78,925 (20) & $3,073(26)$ & 0.161 & $2,961(25)$ & $3,073(26)$ & 0.022 \\
\hline COPD/asthma medication & $29,301(7.1)$ & $28,359(7.1)$ & $942(8.1)$ & 0.039 & $886(7.6)$ & $942(8.1)$ & 0.018 \\
\hline End-stage renal disease & $23,557(5.7)$ & $23,269(5.8)$ & $288(2.5)$ & 0.167 & $270(2.3)$ & $288(2.5)$ & 0.010 \\
\hline Emergency admission & $89,879(22)$ & $85,835(21)$ & $4,044(35)$ & 0.301 & $4,020(35)$ & $4,044(35)$ & 0.004 \\
\hline \multicolumn{8}{|l|}{$\begin{array}{l}\text { Treatments during } \\
\text { hospitalization }\end{array}$} \\
\hline NPPV or respirator use & $12,756(3.1)$ & $11,935(3.0)$ & $821(7.1)$ & 0.188 & $725(6.2)$ & $821(7.1)$ & 0.033 \\
\hline IABP & $6,534(1.6)$ & $6,038(1.5)$ & $496(4.3)$ & 0.165 & $392(3.4)$ & $495(4.3)$ & 0.046 \\
\hline VA-ECMO & $335(0.1)$ & $317(0.1)$ & $18(0.2)$ & 0.022 & $15(0.1)$ & $18(0.2)$ & 0.007 \\
\hline \multicolumn{8}{|l|}{$\begin{array}{l}\text { No. stents implanted at first } \\
\text { PCI }\end{array}$} \\
\hline 0 & $35,182(8.5)$ & $34,165(8.5)$ & $1,017(8.7)$ & 0.018 & $913(7.8)$ & $1,017(8.7)$ & 0.001 \\
\hline 1 & $249,876(60)$ & $242,993(60)$ & $6,883(59)$ & & $7,036(60)$ & $6,883(59)$ & \\
\hline 2 & $100,728(24)$ & $97,825(24)$ & $2,903(25)$ & & $2,919(25)$ & $2,902(25)$ & \\
\hline$\geq 3$ & $28,025(6.8)$ & $27,183(6.8)$ & $842(7.2)$ & & $776(6.7)$ & $842(7.2)$ & \\
\hline CR during hospitalization & $59,867(15)$ & $53,174(13)$ & $6,693(58)$ & 1.044 & $6,686(57)$ & $6,692(58)$ & 0.001 \\
\hline Hospital LOS (days) & $4[3-9]$ & $4[3-9]$ & $7[3-15]$ & 0.225 & $6[3-14]$ & $7[3-15]$ & 0.051 \\
\hline
\end{tabular}

Data are presented as number (\%) or median (interquartile range). sCAD, stable coronary artery disease; VA, venoarterial. Other abbreviations as in Table 1.

\begin{tabular}{|c|c|c|c|c|c|c|}
\hline \multirow[b]{3}{*}{ Primary endpoint } & \multicolumn{3}{|c|}{ ACS group } & \multicolumn{3}{|c|}{ sCAD group } \\
\hline & Adjusted HR & $95 \% \mathrm{Cl}$ & $P$ value & Adjusted HR & $95 \% \mathrm{Cl}$ & $P$ value \\
\hline & & & & & & \\
\hline All-cause mortality & 0.52 & $0.47-0.59$ & $<0.01$ & 0.72 & $0.65-0.80$ & $<0.01$ \\
\hline \multicolumn{7}{|l|}{ Secondary endpoints } \\
\hline Emergency PCl & 0.86 & $0.74-1.00$ & 0.06 & 1.03 & $0.82-1.29$ & 0.80 \\
\hline Planned PCl & 1.01 & $0.96-1.07$ & 0.06 & 1.06 & $0.99-1.13$ & 0.06 \\
\hline Acute heart failure & 0.67 & $0.58-0.78$ & $<0.01$ & 0.76 & $0.67-0.87$ & $<0.01$ \\
\hline Gastrointestinal bleeding & 0.86 & $0.69-1.08$ & 0.19 & 1.14 & $0.92-1.40$ & 0.23 \\
\hline Stroke or $\mathrm{ICH}$ & 0.93 & $0.73-1.20$ & 0.59 & 0.75 & $0.58-0.98$ & 0.04 \\
\hline
\end{tabular}

$\mathrm{Cl}$, confidence interval; $\mathrm{HR}$, hazard ratio; $\mathrm{ICH}$, intracranial hemorrhage. Other abbreviations as in Tables 1,2. 


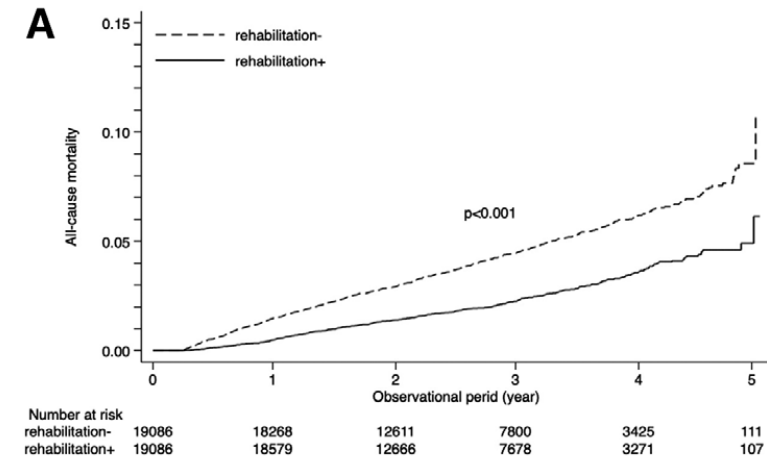

Kaplan-Meier curves for ACS gruop

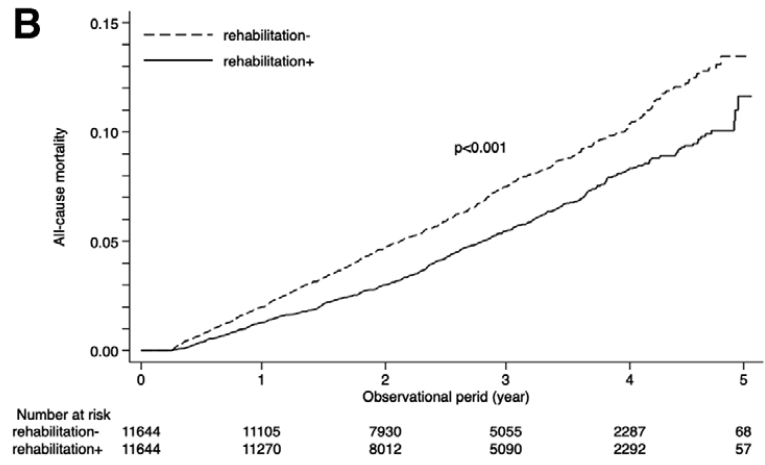

Kaplan-Meier curves for SCAD gruop

Figure 4. Kaplan-Meier curves for patients with and without outpatient cardiac rehabilitation among (A) acute coronary syndrome (ACS) and (B) stable coronary artery disease (SCAD) patients. Outpatient cardiac rehabilitation (solid lines) was associated with better prognosis than no rehabilitation (dotted lines) in both groups.

95\% CI 0.74-1.00; $\mathrm{P}=0.05)$. There was no significant relationship between planned PCI and gastrointestinal bleeding in either the ACS or SCAD group. In addition, when analyses were performed using a multivariable Cox proportional hazard model for all outpatient cohorts, HR for all-cause mortality in patients with outpatient CR was 0.49 (95\% CI $0.44-0.54 ; \mathrm{P}<0.001)$ in the ACS group and $0.72(95 \% \mathrm{CI}$ $0.67-0.78 ; \mathrm{P}<0.001)$ in the $\mathrm{SCAD}$ group. Additional analysis using propensity score matching analysis limited to patients who underwent inpatient CR during the first hospitalization revealed that the adjusted HR for all-cause mortality was $0.52(95 \%$ CI $0.46-0.59 ; \mathrm{P}<0.001)$ in the ACS group and $0.69(95 \%$ CI $0.61-0.79 ; \mathrm{P}<0.001)$ in the sCAD group. The outcomes of the sensitivity analyses revealed consistent results with the primary analysis for all-cause mortality.

\section{Discussion}

The significant findings of this nationwide study using the largest administrative database in Japan were that: (1) participation in outpatient CR was extremely low compared with inpatient $\mathrm{CR}$, and was lower in patients with SCAD than ACS; (2) there were marked regional differences in the prescription rate of CR in Japan; and (3) participation in outpatient CR was associated with lower mortality and hospitalization for acute heart failure. To the best of our knowledge, this is the largest study on the current status of $\mathrm{CR}$ and the efficacy of outpatient CR in Japan.

\section{Rate of Outpatient CR}

The participation rates for inpatient and outpatient CR have been very low in Japan. A previous 2007 study reported that the participation rate of inpatient $\mathrm{CR}$ in patients with ACS was 20\% in Japanese Circulation Society-certified teaching hospitals, whereas the nationwide participation rate in outpatient CR after ACS was estimated to be only $3.8-7.6 \%{ }^{14}$ Although the regional clinical alliance path for bridging acute-to-chronic phase treatment was available for patients with ACS, the rate of implementation of the regional clinical alliance path was only $10 \%$ in 2009 , which was lower than that in stroke. ${ }^{15}$ In 2018, the annual report from the JROAD database reported that the number of new patients taking part in $\mathrm{CR}$ and the number of facilities providing CR were increasing. ${ }^{9}$ In the present study, there were gradual annual increases in the rate of CR participation. However, the participation rates for outpatient CR were very low (9\% for patients with ACS and 3\% for patients with sCAD) compared with inpatient $\mathrm{CR}$. The rate of CR participation is also low worldwide. Participation in $\mathrm{CR}$ is a Class Ia recommendation of the American Heart Association/American College of Cardiology guidelines for individuals with ACS or $\mathrm{SCAD},{ }^{2}$ but CR participation rates in the US range from $19 \%$ to $34 \% .^{16,17}$

There may be some reasons explaining the lower rate of outpatient CR participation in Japan. In the present study there were significant regional differences in the rate of $\mathrm{CR}$ participation, and this may be because the number of hospitals approved to provide CR and the number of medical staff may be insufficient in prefectures with lower CR participation rates. The number of hospitals with outpatient CR programs is lower in Japan than in US. ${ }^{18}$ In the present study, the median length of hospital stay was 13 days for ACS patients and 4 days for sCAD patients, which is shorter than the median length of hospital stay for these patient groups in the past 2 decades. ${ }^{19}$ Because of the shorter hospital stay, there is an urgent need for hospitals with an outpatient CR program. If the number of outpatient CR programs in acute care hospitals is not sufficient, outpatient CR programs should be made widely available in smaller hospitals or community clinics.

\section{Effect of Outpatient CR}

We found that attending outpatient $\mathrm{CR}$ was associated with a significant reduction in all-cause mortality. These results are consistent with previously published cohort studies in the US, where Goel et al reported a $45-47 \%$ decrease in all-cause mortality in patients who participated in $\mathrm{CR}$ after $\mathrm{PCI},{ }^{20}$ and Witt et al reported a $56 \%$ decrease in all-cause mortality associated with CR after ACS. ${ }^{17} \mathrm{~A}$ population-based study in Germany found that CR was associated with a lower mortality HR of 0.65 for all-cause mortality in patients with ACS, patients who underwent PCI, and patients who underwent cardiovascular surgery. ${ }^{21}$ A recent systematic review by Anderson et al found a lower 
risk of cardiovascular death following acute myocardial infarction or revascularization in patients participating in CR. ${ }^{3}$ Evidence supporting outpatient $\mathrm{CR}$ for patients with SCAD is weak compared with that for patients with ACS. A 2018 systematic review of 7 randomized control trials in SCAD patients found no evidence of benefit in terms of all-cause mortality..$^{22} \mathrm{~A}$ possible explanation for the apparent discrepancy with the results of the present study is that the sample size of the systematic review was relatively small and included patients with SCAD without PCI. In the present study, outpatient $\mathrm{CR}$ was associated with lower mortality and acute heart failure in both the ACS and sCAD groups. The greater survival benefits in the present study may be explained by the comprehensive effects of outpatient CR. Patients with outpatient CR underwent not only exercisebased interventions, but also a combination of medical interventions (e.g., lifestyle behavior changes, risk factor modification, guideline-based medications, and close follow-up at specialized facilities). Kamiya et al reported that the multidisciplinary team approach, including exercise and patient education, is effective in patients with heart failure. ${ }^{23}$ The combination of interventions must have resulted in higher motivation and better self-management skills, and consequently a reduction in the risk of all-cause mortality and acute heart failure.

In this study we reported all-cause mortality based on the logic-based method because the original mortality definition in the NDB was not specified. Although the nationwide long-term mortality in patients with sCAD with outpatient CR has never been reported in Japan, the crude mortality in patients with ACS was almost similar to that in other registries. ${ }^{24}$ It should be noted that the crude mortality in patients with ACS was slightly lower than in patients with SCAD. One of the possible reasons for this is that patients with SCAD were older than those with ACS and may have had several comorbidities, such as arrhythmia, heart failure, stroke, and peripheral artery disease..$^{25}$

Similar to previous reports from the US and Europe, ${ }^{17,20,21}$ the present study from Japan demonstrated that CR reduces mortality. In contrast, the findings suggested that outpatient CR participation remained low and there was marked regional variation in CR participation in Japan. Strategies to increase CR by expanding the number of certified facilities and educated medical staff are needed, and we should examine the effects of these strategies in the future.

\section{Study Limitations}

Although this study used a nationwide real-world cohort, it has several limitations. First, this was a retrospective observational study using administrative data and unmeasured confounders were present, such as cardiac function, baseline activities of daily living before admission or at discharge, the extent or duration of CR, details of the CR program, and culprit lesions in patients with ACS. In this study we performed multivariate Cox regression analysis in addition to propensity matching and demonstrated the robustness of the study. Second, the definitions of each outcome, such as acute heart failure and stroke, are not fully validated. Third, the causes of mortality are not known. Therefore, we may have included accidental deaths, and the findings may not reflect exact cardiovascular outcomes. However, the number of accidental deaths is usually very low and would have been unlikely to affect the analyses. Finally, the database did not include a small number of patients who were not covered by medical insurance.

\section{Conclusions}

In Japan, the prescription rate for outpatient CR after PCI was very low, with even more striking differences in some regions. Outpatient CR was associated with beneficial outcomes in patients with ACS and SCAD.

\section{Acknowledgments}

The authors are grateful to Tsuneyuki Higashino and all the investigators at Mitsubishi Research Institute (Tokyo, Japan) for data preparation and management.

\section{Sources of Funding}

This research was funded by a Labor Research Grant (Grant no. 19FA1002) from the Ministry of Health, Labour and Welfare, Japan.

\section{Disclosures}

The authors have no conflicts of interest to declare.

\section{IRB Information}

This study was approved by the Ethics Committee of Nara Medical University (Registration no. 1123-2).

\section{Data Availability}

The deidentified participant data will not be shared.

\section{References}

1. O'Gara PT, Kushner FG, Ascheim DD, Casey DE, Chung MK, de Lemos JA, et al. 2013 ACCF/AHA guideline for the management of ST-elevation myocardial infarction: A report of the American College of Cardiology Foundation/American Heart Association Task Force on Practice Guidelines. Circulation 2013; 127: e362-e425.

2. Smith SC Jr, Benjamin EJ, Bonow RO, Braun LT, Creager MA, Franklin BA, et al. AHA/ACCF secondary prevention and risk reduction therapy for patients with coronary and other atherosclerotic vascular disease: 2011 update: A guideline from the American Heart Association and American College of Cardiology Foundation endorsed by the World Heart Federation and the Preventive Cardiovascular Nurses Association. $J$ Am Coll Cardiol 2011; 58: 2432-2446.

3. Anderson L, Oldridge N, Thompson DR, Zwisler AD, Rees K, Martin N, et al. Exercise-based cardiac rehabilitation for coronary heart disease: Cochrane systematic review and meta-analysis. $J$ Am Coll Cardiol 2016; 67: 1-12.

4. JCS Joint Working Group. Guidelines for rehabilitation in patients with cardiovascular disease (JCS 2012): Digest version. Circ J 2014; 78: 2022-2093.

5. Schopfer DW, Krishnamurthi N, Shen H, Duvernoy CS, Forman DE, Whooley MA. Association of veterans health administration home-based programs with access to and participation in cardiac rehabilitation. JAMA Intern Med 2018; 178: 715-717.

6. Beatty AL, Truong M, Schopfer DW, Shen H, Bachmann JM, Whooley MA. Geographic variation in cardiac rehabilitation participation in Medicare and Veterans Affairs populations: Opportunity for improvement. Circulation 2018; 137: 1899-1908.

7. Goto Y. Current state of cardiac rehabilitation in Japan. Prog Cardiovasc Dis 2014; 56: 557-562.

8. Kohzuki M. Late phase II cardiac rehabilitation, which is adding life to years and years to life, is still underused in Japan. Circ $J$ 2016; 80: $1697-1699$.

9. Japanese Registry Of All cardiac and vascular Diseases (JROAD). Survey on medical cardiovascular disease 2018. http://www.j-circ. or.jp/jittai_chosa/jittai_chosa2018web.pdf [in Japanese].

10. Yasunaga H. Real world data in Japan: Chapter I NDB. Ann Clin Epidemiol 2019; 1: 28-30.

11. Kubo S, Noda T, Myojin T, Nishioka Y, Higashino T, Matsui H, et al. National Database of Health Insurance Claims and Specific Health Checkups of Japan (NDB): Outline and patient-matching 
technique. bioRxiv, doi:10.1101/280008.

12. Noda T, Kubo S, Myojin T, Nishioka Y, Higashino T, Matsui $\mathrm{H}$, et al. Patient-matching technique in National Database of Health Insurance Claims and Specific Health Checkups of Japan (NDB). J Health Welfare Statistics 2017; 64: 7-13 (in Japanese).

13. Kubo S, Noda T, Nishioka Y, Myojin T, Nakanishi Y, Furihata $\mathrm{S}$, et al. Mortality tracking using the National Database of Health Insurance Claims and Specific Health Checkups of Japan (NDB). Jpn J Med Informatics 2019; 39: 297-301 (in Japanese).

14. Goto Y, Saito M, Iwasaka T, Daida H, Kohzuki M, Ueshima K, et al. Poor implementation of cardiac rehabilitation despite broad dissemination of coronary interventions for acute myocardial infarction in Japan: A nationwide survey. Circ J 2007; 71: $173-$ 179.

15. Arakawa T, Kumasaka L, Nakanishi M, Nagayama M, Adachi $\mathrm{H}$, Ikeda $\mathrm{K}$, et al. Regional clinical alliance path and cardiac rehabilitation after hospital discharge for acute myocardial infarction patients in Japan: A nationwide survey. Circ J 2016; 80: $1750-1755$.

16. Ades PA, Keteyian SJ, Wright JS, Hamm LF, Lui K, Newlin K, et al. Increasing cardiac rehabilitation participation from $20 \%$ to $70 \%$ : A road map from the Million Hearts Cardiac Rehabilitation Collaborative. Mayo Clin Proc 2017; 92: 234-242.

17. Witt BJ, Jacobsen SJ, Weston SA, Killian JM, Meverden RA, Allison TG, et al. Cardiac rehabilitation after myocardial infarction in the community. $J$ Am Coll Cardiol 2004; 44: 988-996.

18. Curnier DY, SavagePD, Ades PA. Geographic distribution of cardiac rehabilitation programs in the United States. J Cardiopulm Rehab 2005; 25: 80-84.

19. Kinjo K, Sato H, Nakatani D, Mizuno D, Shimizu M, Hishida E, et al. Predictors of length of hospital stay after acute myocardial infarction in Japan. Circ J 2004; 68: 809-815.

20. Goel K, Lennon RJ, Tilbury RT, Squires RW, Thomas RJ. Impact of cardiac rehabilitation on mortality and cardiovascular events after percutaneous coronary intervention in the community. Circulation 2011; 123: 2344-2352.

21. de Vries H, Kemps HM, van Engen-Verheul MM, Kraaijenhagen RA, Peek N. Cardiac rehabilitation and survival in a large representative community cohort of Dutch patients. Eur Heart $J$ 2015; 36: $1519-1528$

22. Long L, Anderson L, Dewhirst AM, He J, Bridges C, Gandhi M, et al. Exercise-based cardiac rehabilitation for adults with stable angina. Cochrane Database Syst Rev 2018; 2: CD012786.

23. Kamiya K, Sato Y, Takahashi T, Tsuchihashi-Makaya M, Kotooka N, Ikegame T, et al. Multidisciplinary cardiac rehabilitation and long-term prognosis in patients with heart failure. Circ Heart Fail 2020; 13: e006798.

24. Ishihara M, Nakao K, Ozaki Y, Kimura K, Ako J, Noguchi T, et al. Long-term outcomes of non-ST-elevation myocardial infarction without creatine kinase elevation: The J-MINUET study. Circ J 2017; 81: 958-965.

25. Soeda T, Okura H, Saito Y, Nakao K, Ozaki Y, Kimura K, et al. Clinical characteristics and in-hospital outcomes in patients aged 80 years or over with cardiac troponin-positive acute myocardial infarction: J-MINUET study. J Cardiol 2021; 77: $139-146$.

\section{Supplementary Files}

Please find supplementary file(s);

http://dx.doi.org/10.1253/circrep.CR-20-0143 